\title{
A nonlinear technique for the analysis of plasmid instability in micro-organisms
}

\author{
A. Michael Davidson, ${ }^{1}$ Andrew Dunn, ${ }^{2 *}$ Martin J. Day ${ }^{2}$ and Peter F. Randerson ${ }^{2}$ \\ Departments of ${ }^{1}$ Mathematics and ${ }^{2}$ Pure and Applied Biology, University of Wales College of Cardiff, PO Box 915 , \\ Cardiff CF1 3TL, UK
}

(Received 27 February 1989; revised 19 June 1989; accepted 16 October 1989)

\begin{abstract}
A nonlinear technique to calculate parameters for segregational instability and differences in cellular growth-rate for plasmid-bearing micro-organisms growing in batch or continuous culture is presented. This method is compared with an approximate technique based upon linear regression. The accuracy and sensitivity of the results are evaluated by use of simulated data and biological data taken from experiments with Pseudomonas aeruginosa(pGSS15) and Escherichia coli(pHSG415). It is demonstrated that the nonlinear analysis gives results which are significantly more accurate and which show much better agreement with the data. Consequently, the new analysis leads to quite different conclusions with regard to the nature of the instability of the plasmid-bearing strain. This method offers an opportunity to study the genetic and physiological aspects of plasmid instability and so aid the design and optimization of cloning vectors.
\end{abstract}

\section{Introduction}

Mathematical models which describe the population dynamics of non-conjugative plasmid-bearing microorganisms growing in batch or continuous culture have been used by many authors (e.g. Cooper et al., 1987; Dunn et al., 1988; Lenski \& Bouma, 1987; Stewart \& Levin, 1977). The model considered here, which is discussed in detail below, is based on the equations proposed by Stewart \& Levin (1977). It gives the variation with time of the concentrations of plasmidbearing and plasmid-free cells and is dependent upon three parameters, viz. the growth-rate of plasmidbearing cells, the growth rate of plasmid-free cells and the rate of loss of the plasmid genotype. The last comprises principally plasmid segregation and mutation. If these parameters are assumed to have constant values with respect to time, the mathematical model can be solved explicitly and the solution depends upon the difference in growth rates rather than the individual values. The purpose of this paper is to evaluate a method by which the segregation and cellular growth rate difference parameters may be deduced by fitting the predictions of the model to experimental data.

One approach to this was proposed by Cooper $e t$ al. (1987), who identified three regimes of behaviour depending upon the relative values of the two para-

\footnotetext{
* Author for correspondence (electronic-mail address: DUNNA(UK.AC.CARDIFF)
}

meters. They provided exponential approximations to part of the model solution for these three cases, which could then be fitted to appropriate data by a standard linear regression technique. However, the parameter values given by this technique can only be approximate because the model itself is nonlinear. For this reason, it is desirable to fit the true model solution to the data: this was accomplished using the nonlinear least-squares fitting procedure presented in this paper.

\section{Methods}

Numerical calculations were done on a DEC VAX 11-780 using the NAG (Numerical Algorithms Group) Fortran routines (Fox, 1987) E04JBF (quasi-Newton minimization) and F01ABF (matrix inversion). A key to the various symbols used in this paper is given in an appendix.

\section{The Mathematical Model}

If $x_{+}$and $x_{-}$represent the concentrations of plasmidbearing and plasmid-free cells respectively growing in continuous culture with dilution rate $D$, then the mathematical model describing the change in the density of each population with time is

$$
\begin{aligned}
& \frac{\mathrm{d}}{\mathrm{d} t} x_{+}=\mu_{+} x_{+}-R x_{+}-D x_{+} \\
& \frac{\mathrm{d}}{\mathrm{d} t} x_{-}=\mu_{-} x_{-}+R x_{+}-D x_{-}
\end{aligned}
$$


(Stewart \& Levin, 1977) where $\mu_{+}$and $\mu_{-}$are the growth rates of $x_{+}$and $x_{-}$, and $R$ is the plasmid segregation rate. It is usual to work, not with the actual values of $x_{+}$and $x_{-}$, but to express them as proportions of the total cell population, i.e.

$$
p_{+}=\frac{x_{+}}{x_{+}+x_{-}} \text {and } p_{-}=\frac{x_{-}}{x_{+}+x_{-}}
$$

where $p_{+}+p_{-}=1$. If the total cell concentration $\left(x_{+}+x_{-}\right)$is assumed to be constant, then equations (1) hold for $p_{+}$and $p_{-}$and can be rearranged to yield a single equation describing plasmid instability in both batch and continuous culture

$$
\frac{\mathrm{d}}{\mathrm{d} t} p_{+}=\mathrm{d} \mu p_{+}^{2}-(\mathrm{d} \mu+R) p_{+}
$$

(Cooper et al., 1987) where $\mathrm{d} \mu=\mu_{-}-\mu_{+}$. It should be noted that the same equation holds even if the total cell concentration is variable with respect to time. This will occur during batch growth, or during continuous culture if $\mathrm{d} \mu$ is not constant or if the nutrient yields of each cell type are not equal (Dunn et al., 1988).

From equation (3) it can be seen that the behaviour of $p_{+}$depends only on $\mathrm{d} \mu$ and $R$ rather than on the individual growth rates $\mu_{+}$and $\mu_{-}$. If the further assumptions that $\mathrm{d} \mu$ and $R$ are constant are made, equation (3) can be solved and for the case $\mathrm{d} \mu+R \neq 0$

$$
p_{+}(t)=\frac{p_{+0}(\mathrm{~d} \mu+R)}{\left(p_{-0} \mathrm{~d} \mu+R\right) \mathrm{e}^{(\mathrm{d} \mu+R) t}+\mathrm{d} \mu p_{+0}}
$$

(this is equivalent to the equation of Lenski \& Bouma, 1987) where $p_{+0}$ and $p_{-0}$ are the values of $p_{+}$and $p_{-}$at $t=0$. For the case $\mathrm{d} \mu+R=0$

$$
p_{+}(t)=\frac{p_{+0}}{1-p_{+0} \mathrm{~d} \mu t}
$$

It can be seen from equation (3) that the equilibrium values of $p_{+}$as $t \rightarrow \infty$ are

$$
\tilde{p}_{+}=0 \quad \text { or } \quad \tilde{p}_{+}=1+\frac{R}{\mathrm{~d} \mu}
$$

Since $0 \leqslant p_{+} \leqslant 1$ and $R \geq 0$, the second possibility can only occur when $\mathrm{d} \mu<0$ and $|\mathrm{d} \mu|>R$. In all other cases the solution will tend to $p_{+}=0$. The corresponding equilibria for $p_{-}$are

$$
\tilde{p}_{-}=1 \quad \text { or } \quad \tilde{p}_{-}=-\frac{R}{\mathrm{~d} \mu}
$$

\section{The Curve-fitting Techniques}

\section{Linear regression method}

The fitting procedure of Cooper et al. (1987) considers three different types of behaviour and in each case approximates equation (4) by an exponential relation- ship from which the parameters can be identified by linear regression:

(a) $\mathrm{d} \mu \gg R$ when $p_{-} \propto \mathrm{e}^{\mathrm{d} \mu t}$ for small values of $t$;

(b) $\mathrm{d} \mu \leqslant R$ when $p_{+} \propto \mathrm{e}^{-(\mathrm{d} \mu+R) t}$ for large values of $t$;

(c) $\mathrm{d} \mu<0$ and $|\mathrm{d} \mu| \gg R$ when $\left(p_{+}-\tilde{p}_{+}\right) \propto \mathrm{e}^{(\mathrm{d} \mu+R) t}$ for large values of $t$.

For case $(c)$ the equilibrium value $\tilde{p}_{+}$, which is the value of $p_{+}$as $t \rightarrow \infty$, is needed, and this must be taken from the data. In each case, regressing $\log _{\mathrm{e}} p_{+}$or $\log _{\mathrm{e}} p_{-}$against $t$ enables the values for $\mathrm{d} \mu$ and $R$ to be estimated.

Numerical results for cases $(a)$ and $(b)$ were presented by Cooper et al. (1987) and appear to show an excellent fit between the observed data points and the model solution. However, the curve given is the exponential approximation and the fit is simply a measure of the goodness of fit of the regression line. The agreement is less good if the more meaningful comparison is made between the data points and the solution to equation (3) using the estimated parameter values. This is illustrated in Fig. 1 for cases $(a)$ and $(b)$ using data points generated by equation (4) with known parameter values. It can be observed that the fit is reasonable only in the approximately exponential region [i.e. small values of $t$ for case $(a)$ and large values of $t$ for case $(b)]$ and so the parameter values estimated do not describe the complete behaviour. Table 1 gives a comparison between the actual values and those obtained using the linear regression. Note that the actual parameter values fall outside the $95 \%$ confidence limits because the model solution is not truly exponential.

\section{Nonlinear fitting method}

The linear method relies upon the assumption that a section of the trajectory is exponential and this leads to the intrinsic error already demonstrated. The nonlinear procedure is exact because the data points are fitted to the true solution of equation (3). This was accomplished by calculating initially the trajectory for $p_{+}(t)$ using approximated values of $\mathrm{d} \mu, R$ and $p_{+0}$. The error was then measured as the sum of squared differences, $S$, between the model solution $p_{+}\left(t_{i}\right)$ and each data point $\bar{p}_{+}\left(t_{i}\right)$, i.e.

$$
S=\sum_{i=1}^{N}\left\{p_{+}\left(t_{i}\right)-\bar{p}_{+}\left(t_{i}\right)\right\}^{2}
$$

where $N$ is the number of data points. The parameter values were updated subsequently using a suitable minimization algorithm until the error criterion reached a minimum. The algorithm used was a quasi-Newton method with simple bounds on the variables. This prevents the possibility of non-physical parameter 


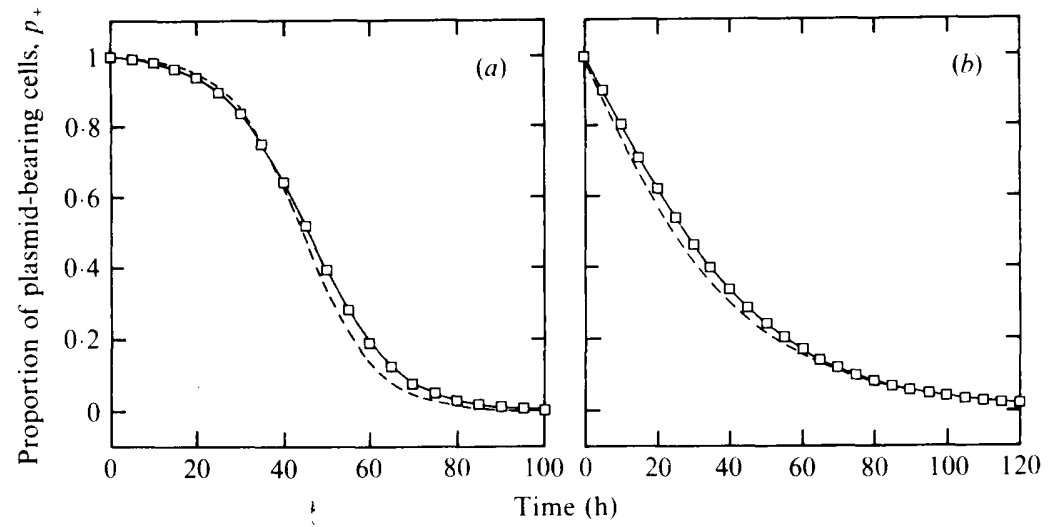

Fig. 1. Comparison between actual solutions to equation (3) and the solutions predicted by the two fitting methods. (a) $\mathrm{d} \mu=1.00 \times 10^{-1}$ and $R=1.00 \times 10^{-3} ;(b) \mathrm{d} \mu=2.00 \times 10^{-2}$ and $R=2.00 \times 10^{-2} . \square$, Actual solution; 一, solution predicted by nonlinear method; ---, solution predicted by linear method

Table 1. Comparison between the two fitting techniques in analysis of the solution to equation (3)

\begin{tabular}{|c|c|c|c|c|c|c|}
\hline \multirow[b]{2}{*}{ Case } & \multirow[b]{2}{*}{ Parameter } & \multirow[b]{2}{*}{$\begin{array}{l}\text { Actual value } \\
\qquad\left(h^{-1}\right)\end{array}$} & \multicolumn{2}{|r|}{ Linear method } & \multicolumn{2}{|c|}{ Nonlinear method } \\
\hline & & & $\begin{array}{c}\text { Predicted value } \\
\qquad\left(h^{-1}\right)\end{array}$ & $\begin{array}{l}95 \% \text { Confidence limits } \\
\left(\mathrm{h}^{-1}\right)\end{array}$ & $\begin{array}{l}\text { Predicted value } \\
\qquad\left(\mathrm{h}^{-1}\right)\end{array}$ & $\begin{array}{l}95 \% \text { Confidence limits } \\
\left(\mathrm{h}^{-1}\right)\end{array}$ \\
\hline \multirow[t]{2}{*}{ (a) } & $\mathrm{d} \mu$ & $1.00 \times 10^{-1}$ & $1.17 \times 10^{-1}$ & $1.13 \times 10^{-1}$ to $1.21 \times 10^{-1}$ & $1.00 \times 10^{-1}$ & $1.00 \times 10^{-1}$ to $1.00 \times 10^{-1}$ \\
\hline & $R$ & $1.00 \times 10^{-3}$ & $6.40 \times 10^{-4}$ & $5.70 \times 10^{-4}$ to $7.10 \times 10^{-4}$ & $1.00 \times 10^{-3}$ & $1.00 \times 10^{-3}$ to $1.00 \times 10^{-3}$ \\
\hline \multirow[t]{2}{*}{ (b) } & $\mathrm{d} \mu$ & $2.00 \times 10^{-2}$ & $1.22 \times 10^{-2}$ & $1.11 \times 10^{-2}$ to $1.34 \times 10^{-2}$ & $2.00 \times 10^{-2}$ & $2.00 \times 10^{-2}$ to $2.00 \times 10^{-2}$ \\
\hline & $R$ & $2.00 \times 10^{-2}$ & $2.50 \times 10^{-2}$ & $2.37 \times 10^{-2}$ to $2.63 \times 10^{-2}$ & $2.00 \times 10^{-2}$ & $2.00 \times 10^{-2}$ to $2.00 \times 10^{-2}$ \\
\hline
\end{tabular}

values, such as $R<0$ or $p_{+0}>1$, which were found to be a problem with certain data sets.

A weighting factor, such as the angular transformation (Sokal \& Rolf, 1969), may be included in the above expression in order to homogenize the binomially distributed errors inherent in the experimental data. This error distribution results as a consequence of sampling a finite number of cells at each data point for the presence of the plasmid, so that the standard deviation (SD) of each reading is given by

$$
\mathrm{SD}=\left\{\bar{p}_{+}\left(t_{\mathrm{i}}\right) \bar{p}_{-}\left(t_{\mathrm{i}}\right) \frac{1}{n}\right\}^{1 / 2}
$$

where $n$ is the number of cells sampled.

The variance of each parameter value was calculated according to the formula

$$
\operatorname{var} v_{\mathrm{i}}=\frac{2 S}{N-3} \boldsymbol{H}_{\mathrm{ij}}
$$

(Fox, 1987) where $v=\left(p_{+0}, R, \mathrm{~d} \mu\right)^{T}$ and $H$ is the inverse of the Hessian matrix of second partial derivatives, i.e.

$$
\boldsymbol{H}=\boldsymbol{G}^{-1}
$$

where

$$
G_{\mathrm{ij}}=\frac{\delta^{2} S}{\delta v_{\mathrm{i}} \delta v_{\mathrm{j}}}
$$

The $100(1-\beta)$ confidence interval on $v$ is given by

$$
v_{i} \pm t_{(\beta / 2, N-3)} \cdot\left(\operatorname{var} v_{\mathrm{i}}\right)^{1 / 2}
$$

where $t_{(\beta / 2, N-3)}$ is the $100 \beta / 2$ percentage point of the $t$ distribution with $N-3$ degrees of freedom. When fitted to data generated by equation (4), the values of $\mathrm{d} \mu, R$ and $p_{+0}$ had the same order of accuracy as the actual data (Table 1 and Fig. 1).

\section{Robustness and sensitivity of the solutions}

The question of the robustness of the curve fitting technique arises due to experimental errors which are inherent in the data values. Such errors mean that, even if the mathematical model is correct, it will not be possible to find a solution to fit the data exactly. In order to illustrate this, both fitting methods were applied to a known solution of equation (3) which was perturbed using binomially distributed errors assuming 200 trials. This was equivalent to sampling 200 cells for the presence of the plasmid. The same number of data points were analysed by each method. These results, which are given in Fig. 2 and Table 2, show the nonlinear method to be extremely robust. 


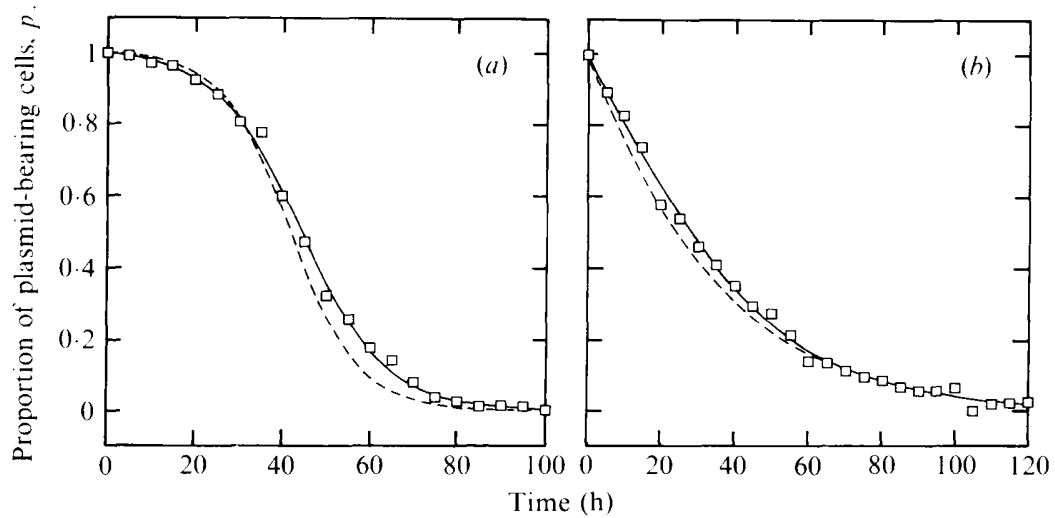

Fig. 2. Comparison between the two fitting techniques in analysis of the perturbed solution to equation (3). (a) $\mathrm{d} \mu=1.00 \times 10^{-1}$ and $R=1.00 \times 10^{-3} ;(b) \mathrm{d} \mu=2.00 \times 10^{-2}$ and $R=2.00 \times 10^{-2} . \square$, Data points; - , solution predicted by nonlinear method; $-\cdots--$, solution predicted by linear method.

Table 2. Comparison between the two fitting methods in analysis of the perturbed solution to equation (3)

\begin{tabular}{|c|c|c|c|c|c|c|}
\hline \multirow[b]{2}{*}{ Case } & \multirow[b]{2}{*}{ Parameter } & \multirow[b]{2}{*}{$\begin{array}{l}\text { Actual value } \\
\qquad\left(\mathrm{h}^{-1}\right)\end{array}$} & \multicolumn{2}{|r|}{ Linear method } & \multicolumn{2}{|c|}{ Nonlinear method } \\
\hline & & & $\begin{array}{l}\text { Predicted value } \\
\qquad\left(h^{-1}\right)\end{array}$ & $\begin{array}{l}95 \% \text { Confidence limits } \\
\left(\mathrm{h}^{-1}\right)\end{array}$ & $\begin{array}{l}\text { Predicted value } \\
\qquad\left(\mathrm{h}^{-1}\right)\end{array}$ & $\begin{array}{l}95 \% \text { Confidence limits } \\
\left(\mathrm{h}^{-1}\right)\end{array}$ \\
\hline \multirow[t]{2}{*}{ (a) } & $\mathrm{d} \mu$ & $1.00 \times 10^{-1}$ & $1.27 \times 10^{-1}$ & $8.73 \times 10^{-2}$ to $1.67 \times 10^{-1}$ & $1.01 \times 10^{-1}$ & $9.53 \times 10^{-2}$ to $1.07 \times 10^{-1}$ \\
\hline & $R$ & $1.00 \times 10^{-3}$ & $6.15 \times 10^{-4}$ & $2.11 \times 10^{-4}$ to $1.62 \times 10^{-3}$ & $9.92 \times 10^{-4}$ & $7.24 \times 10^{-4}$ to $1.26 \times 10^{-3}$ \\
\hline \multirow[t]{2}{*}{ (b) } & $\mathrm{d} \mu$ & $2.00 \times 10^{-2}$ & $9.55 \times 10^{-3}$ & $8.39 \times 10^{-3}$ to $1.07 \times 10^{-2}$ & $2.01 \times 10^{-2}$ & $1.57 \times 10^{-2}$ to $2.45 \times 10^{-2}$ \\
\hline & $R$ & $2.00 \times 10^{-2}$ & $2.55 \times 10^{-2}$ & $2.42 \times 10^{-2}$ to $2.69 \times 10^{-2}$ & $1.96 \times 10^{-2}$ & $1.80 \times 10^{-2}$ to $2.12 \times 10^{-2}$ \\
\hline
\end{tabular}

Model sensitivity refers to the fact that very different parameter values can give quite similar trajectories. This effect can be observed in Tables $1-3$ and Figs $1-3$. These serve to illustrate the importance of using the whole trajectory as in the nonlinear technique.

\section{Analysis of biological data}

The high level of accuracy of the nonlinear method in analysing simulated data has been demonstrated. The closeness to which the model solution fits biological data will depend upon the validity of the model itself. Fig. 3 and Table 3 show the results of an analysis of two such data sets. These are (a) Pseudomonas aeruginosa PAOl(pGSS15) (Sharpe, 1984) growing at $30^{\circ} \mathrm{C}$ in glucose minimal medium at a dilution rate of $0.04 \mathrm{~h}^{-1}$ (Lewington, 1987) and (b) Escherichia coli R V308(pHSG415) (Hashimoto-Gotoh et al., 1981) growing at $30^{\circ} \mathrm{C}$ in glucose minimal medium at a dilution rate of $0.304 \mathrm{~h}^{-1}$ (Caulcott et al., 1987). Both strains were grown in nonselective medium. In contrast with the linear method, the solutions predicted by the nonlinear analysis show extremely good agreement with the data.

\section{Discussion}

Our results demonstrate that the linear method of Cooper et al. (1987) is reasonably accurate in analysing unperturbed simulated data (Fig. 1 and Table 1), but that the inclusion of sampling errors in the data reduces considerably the accuracy of the results (Fig. 2 and Table 2). In the analysis of biological data, the linear method gives a very poor fit between the model solution and the data points (Fig. 3 and Table 3). Moreover, this method gives confidence limits on the parameter values which are of questionable worth (Table 1). The parameter values and confidence limits predicted by the nonlinear method, however, give model solutions which show very good agreement with all of the data sets analysed in this paper. This is because the nonlinear method fits the exact model solution to all of the data points; it is thus extremely robust and is not affected significantly by the low sensitivity of the model. It is also more flexible than the linear method in that it can be used to analyse data for all possible values of $\mathrm{d} \mu$ and $R$. It is important to realise that both methods give rate parameter values which are constant with respect to time and so they are 

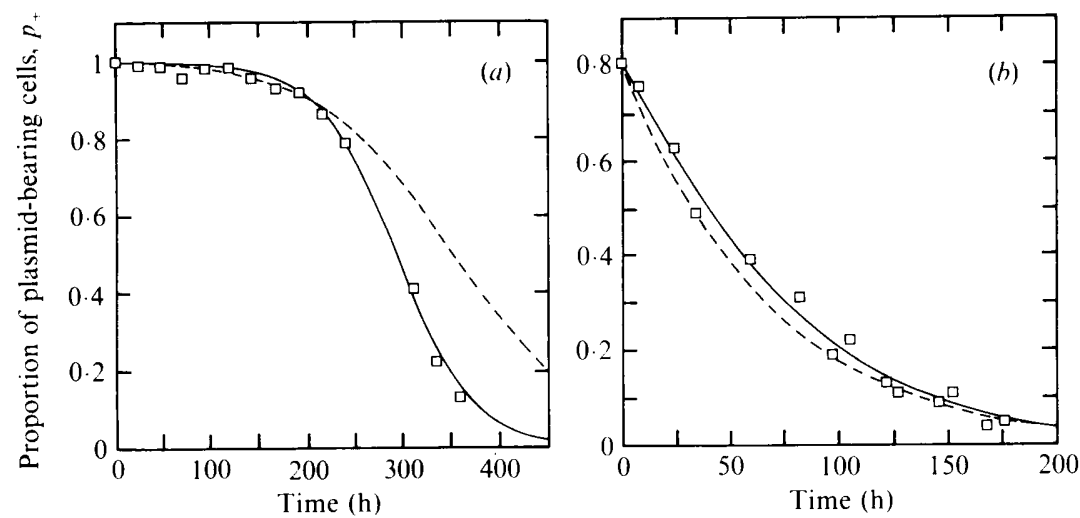

Fig. 3. Solutions predicted by the two fitting methods in analysis of biological data. (a) P. aeruginosa(pGSS15); (b) E. coli(pHSG415). $\square$, Data points; - , solution predicted by nonlinear method; ---, solution predicted by linear regression.

Table 3. Comparison between the two fitting methods in analysis of the biological data

\begin{tabular}{|c|c|c|c|c|c|}
\hline \multirow[b]{2}{*}{ Strain } & \multirow[b]{2}{*}{ Farameter } & \multicolumn{2}{|r|}{ Linear method } & \multicolumn{2}{|c|}{ Nonlinear method } \\
\hline & & $\begin{array}{l}\text { Predicted value } \\
\qquad\left(\mathrm{h}^{-1}\right)\end{array}$ & $\begin{array}{l}95 \% \text { Confidence limits } \\
\left(\mathrm{h}^{-1}\right)\end{array}$ & $\begin{array}{l}\text { Predicted value } \\
\qquad\left(\mathrm{h}^{-1}\right)\end{array}$ & $\begin{array}{l}95 \% \text { Confidence limits } \\
\left(\mathrm{h}^{-1}\right)\end{array}$ \\
\hline (a) $P$. aeruginosa(pGSS15) & $\begin{array}{c}\mathrm{d} \mu \\
R\end{array}$ & $\begin{array}{l}1.40 \times 10^{-2} \\
8.04 \times 10^{-5}\end{array}$ & $\begin{array}{l}7.36 \times 10^{-3} \text { to } 2.06 \times 10^{-2} \\
1.49 \times 10^{-5} \text { to } 3.36 \times 10^{-4}\end{array}$ & $\begin{array}{l}2.52 \times 10^{-2} \\
0.00\end{array}$ & $\begin{array}{l}2.33 \times 10^{-2} \text { to } 2.70 \times 10^{-2} \\
0.00 \quad \text { to } 0.00\end{array}$ \\
\hline (b) E. coli(pHSG415) & $\begin{array}{c}\mathrm{d} \mu \\
R\end{array}$ & $\begin{array}{l}1.67 \times 10^{-3} \\
1.44 \times 10^{-2}\end{array}$ & $\begin{array}{l}5.70 \times 10^{-4} \text { to } 3.25 \times 10^{-3} \\
1.18 \times 10^{-2} \text { to } 1.71 \times 10^{-2}\end{array}$ & $\begin{array}{l}8.46 \times 10^{-3} \\
9.31 \times 10^{-3}\end{array}$ & $\begin{array}{l}5.52 \times 10^{-4} \text { to } 1.64 \times 10^{-2} \\
4.76 \times 10^{-3} \text { to } 1.38 \times 10^{-2}\end{array}$ \\
\hline
\end{tabular}

not suitable for analysing data sets for which $\mathrm{d} \mu$ and $R$ vary greatly. This will occur, for example, where the copy number of the plasmid varies considerably (Jones et al., 1980), or where plasmid or cell mutations arise during the experiment (Helling et al., 1981).

In the analysis of biological data by the two fitting techniques, very different conclusions about the nature of the instability of the plasmid-bearing strain are reached. For example, in their linear analysis of $E$. coli (pHSG415) instability, Caulcott et al. (1987) suggest that plasmid segregation is the dominant factor because they find this parameter to be an order of magnitude greater than the growth-rate difference. However, our analysis shows $\mathrm{d} \mu$ and $R$ to be virtually identical in value (Table 3). Since pHSG415 is a small (7.1 kb), low-copynumber ( $\sim 5$ per chromosome) plasmid which has a partially deleted par sequence (Hashimoto-Gotoh et al., 1981), it would be expected to have the high rate of segregation indicated by both curve-fitting techniques (Seo \& Bailey, 1985; Tucker et al., 1984). For such a plasmid, partitioned randomly at cell division, the nonlinear analysis predicts a theoretical plasmid copynumber of 6.6 per dividing cell (Novick et al., 1975). The comparatively high growth rate difference predicted by the nonlinear analysis may be explained by the presence of constitutively expressed, plasmid-mediated genes for resistance to the antibiotics chloramphenicol, ampicillin and kanamycin. The products of these genes would impose a significant metabolic load on the cell, so reducing the growth rate of the plasmid-bearing strain (Siegel \& Ryu, 1985; Wittrup \& Bailey, 1988).

The nonlinear technique allows the genetic and physiological aspects of plasmid instability in batch or continuous culture to be investigated. It should prove to be an invaluable aid in the design and optimization of plasmid-mediated gene-expression systems.

\section{Appendix: Nomenclature}

\begin{tabular}{|c|c|}
\hline$x_{+}$ & $\begin{array}{l}\text { Plasmid-bearing cell concentration (cells } \\
1^{-1} \text { ) }\end{array}$ \\
\hline$x_{-}$ & Plasmid-free cell concentration (cells $1^{-1}$ ) \\
\hline$p_{+}$ & $\begin{array}{l}\text { Proportion of population comprising } \\
\text { plasmid-bearing cells }\end{array}$ \\
\hline$p_{-}$ & $\begin{array}{l}\text { Proportion of population comprising } \\
\text { plasmid-free cells }\end{array}$ \\
\hline $\begin{array}{l}p_{+0}, p_{-0} \\
\tilde{p}_{+}, \tilde{p}_{-}\end{array}$ & $\begin{array}{l}\text { Values of } p_{+} \text {and } p_{-} \text {at } t=0 \\
\text { Equilibrium values for } p_{+} \text {and } p_{-} \text {cells as } \\
t \rightarrow \infty\end{array}$ \\
\hline
\end{tabular}




$$
\begin{array}{ll}
\bar{p}_{+}, \bar{p}_{-} & \text {Measured values of } p_{+} \text {and } p_{-} \\
\mu_{+} & \text {Plasmid-bearing cell growth rate }\left(\mathrm{h}^{-1}\right) \\
\mu_{-} & \text {Plasmid-free cell growth rate }\left(\mathrm{h}^{-1}\right) \\
\mathrm{d} \mu & \text { Growth rate difference }\left(\mu_{-}-\mu_{+}\right)\left(\mathrm{h}^{-1}\right) \\
R & \text { Plasmid segregation rate }\left(\mathrm{h}^{-1}\right) \\
D & \text { Environmental dilution rate }\left(\mathrm{h}^{-1}\right) \\
t & \text { Time (h) }
\end{array}
$$

A. Dunn gratefully acknowledges the receipt of a University of Wales Studentship.

\section{References}

Caulcott, C. A., Dunn, A., Robertson, H. A., Cooper, N. S., BROWN, M. E. \& RHODES, P. M. (1987). Investigation of the effect of growth environment on the stability of low-copy-number plasmids in Escherichia coli. Journal of General Microbiology 133, 1881-1889.

Cooper, N. S., Brown, M. E. \& Caulcott, C. A. (1987). A mathematical method for analysing plasmid instability in microorganisms. Journal of General Microbiology 133, 1871-1880.

Dunn, A., Davidson, A. M., Day, M. J. \& Randerson, P. F. (1988). A computer analysis of plasmid instability in microorganisms. First International Conference on the Release of Genetically Engineered Microorganisms, Cardiff, 1988, abstract 18, p. 15.

Fox, L. (1987). Numerical Algorithms Group Library Manual Mark 12. Oxford: Numerical Algorithms Group.

Hashimoto-Gotoh, T., Franklin, F. C. H., Nordheim, A. \& Timmis, K. N. (1981). Specific purpose plasmid cloning vectors. 1. Low copynumber, temperature sensitive, mobilization defective, pSC101derived containment vectors. Gene 16, 227-235.
Helling, R. B., KinNey, T. \& Adams, J. (1981). The maintenance of plasmid-containing organisms in populations of Escherichia coli. Journal of General Microbiology 123, 129-141.

Jones, I. M., Primrose, S. B., Robinson, A. \& Ellwood, D. C. (1980) Maintenance of some ColE1-type plasmids in chemostat culture Molecular and General Genetics 180, 579-584.

LeNSK1, R. E. \& Bouma, J. E. (1987). Effects of segregation and selection on the stability of plasmid pACYC184 in Escherichia coli. Journal of Bacteriology 169, 5314-5316.

LEWINGTON, J. (1987). Plasmid instability in pseudomonads. PhD thesis, University of Wales, UK.

Novick, R. P., Wyman, L., Bouanchaud, D. \& Murphy, E. (1975). Plasmid life cycles in Staphylococcus aureus. In Microbiology 1974, pp. 115-129. Edited by D. Schlessinger. Washington, DC: American Society for Microbiology.

Seo, J. H. \& BaIley, J. E. (1985). A segregated model for plasmid content and product synthesis in unstable binary fission recombinant organisms. Biotechnology and Bioengineering 27, 156-165.

SHARPE, G. S. (1984). Broad host range cloning vectors for Gram negative bacteria. Gene 29, 93-102.

SIEGeL, R. \& RYU, D. Y. (1985). Kinetic study of instability of recombinant plasmid pPLc23trpAI in Escherichia coli using twostage continuous culture system. Biotechnology and Bioengineering 27, 28-33.

SOKAL, R. R. \& Rolf, F. J. (1969). Biometry. San Francisco: W. H. Freeman.

Stewart, F. M. \& Levin, B. R. (1977). The population biology of bacterial plasmids: a priori conditions for the existence of conjugationally transmitted factors. Genetics 87, 209-228.

TuCKer, W. T., Miller, C. A. \& COHEN, S. N. (1984). Structural and functional analysis of the par region of the pSC101 plasmid. Cell 38, 191-201.

WitTruP, K. D. \& Bailey, J. E. (1988). A segregated model of recombinant multicopy plasmid propagation. Biotechnology and Bioengineering 31, 304-340. 